\title{
Method of the Year 2009
}

\author{
The ability to return mature body cells to a pluripotent state has wide-ranging \\ potential as a tool for discovery in both disease and basic biology.
}

Our choice for Method of the Year 2009_induced pluripotency as a tool for biological discovery—needs hardly any introduction. Since the first reports of this form of cellular reprogramming a few years ago, research on induced pluripotent stem cells has moved forward amid much attention and at an almost frantic pace. But with the dust of the first heady days settling, with several researchers newly entering the field, and with methods to induce pluripotency becoming more robust, this research is now poised for biological discovery.

In a News Feature on page 17, Monya Baker captures both the excitement and the many challenges ahead, and you can also watch our video. For those who are not entirely familiar with this fast-moving area, we provide a Primer on page 20.

In a Commentary on page 22, Andras Nagy discusses some of the basic questions that research on induced pluripotency could help answer. The nature of pluripotency and the molecular basis of the acquisition and execution of cell fate are subjects at the core of biology and are areas to which this research will undoubtedly contribute. Furthermore, induced pluripotency offers a relatively simple system for studying the reprogramming process itself. The advantages are in part logistical. Unlike research on epigenetic reprogramming by somatic cell nuclear transfer, or on differentiation in embryonic stem cells, research on induced pluripotent stem cells is far more accessible to most biologists.

Induced pluripotent stem cells can be generated from easily available body cells using methods already existing in many labs and, in the case of human research, are not hampered by as much ethical, social or political controversy. However, on page 28, Timothy Caulfield and an international group of collaborators note that this research is not without ethical quandaries of its own and discuss the regulatory policies in place, which are complex and in many cases not designed to meet the specific requirements of the field.

An equally exciting application is the possibility of deriving induced pluripotent stem cells from particular individuals for the study of human disease, as discussed by Lorenz Studer in a Commentary on page 25. This past year has seen the first studies in which human disease phenotypes and drug responses were clearly recapitulated in induced pluripotent stem cell-based in vitro models, and, with facilities being established worldwide for the generation of multiple diseasespecific cell lines, these studies are certainly only the beginning. Furthermore, differentiated derivatives of human induced pluripotent stem cells could conceivably reduce our dependence on cell lines for many basic studies as well.

We also provide on page 34 our annual roundup, necessarily incomplete and somewhat subjective, of Methods to Watch in the years ahead. We are grateful to those readers who took the time to vote for their choice of Method of the Year 2009; visit our blog methagora for more about these votes.

Research on induced pluripotency is still in its early days, and understanding the true potential of these stem cells will require continued investigation and more complete comparisons to embryonic stem cells. Methodological development is still a key part of this process, as is highlighted by several original research papers in this issue and by a Technology Feature on page 44 on methods to make, culture and characterize these cells.

On page 61, Keiichi Fukuda and colleagues report a simple and nongenetic method for purifying cardiomyocytes derived from pluripotent stem cells. Along with better methods for directed differentiation, isolation approaches of this sort are needed to obtain pure preparations of interesting cell types; these will be critical for many applications in both basic and applied research. And mouse tools, too, continue to be improved: in two parallel papers on page 53 and page 56, the laboratories of Konrad Hochedlinger and Rudolf Jaenisch report mouse models in which inducible reprogramming is more robust than with existing tools. On page 6, Jeanne Loring and colleagues emphasize the need for genetically diverse pluripotent cell lines for both basic research and drug screening, a need that may well best be met with induced pluripotent stem cells.

Even a cursory examination of the literature on induced pluripotency over the past 3 years tells an eloquent story. The initial trickle of reports from a handful of laboratories has burgeoned rapidly to a river in full spate. There are twists and turns to be negotiated, and there are almost certainly rocks ahead, but with some foresight and judicious steering there is also the possibility of reaching biological terrain that has remained until now unseen. 